\title{
EXPLICIT BOND OPTION AND SWAPTION FORMULA IN HEATH-JARROW-MORTON ONE FACTOR MODEL
}

\author{
MARC HENRARD
}

\begin{abstract}
We present an explicit formula for European options on coupon bearing bonds and swaptions in the Heath-Jarrow-Morton (HJM) one factor model with non-stochastic volatility. The formula extends the Jamshidian formula for zero-coupon bonds. We provide also an explicit way to compute the hedging ratio $(\Delta)$ to hedge the option with its underlying.
\end{abstract}

\section{INTRODUCTION}

The framework of this article is a Heath-Jarrow-Morton [3] one factor model. The volatility used is non-stochastic and generalizes Ho and Lee [4] and Hull and White [5] volatilities models. In this framework we provide an explicit formula for coupon-bearing bonds and swaptions.

The nature of the situation we want to model being infinite dimensional (we model the whole interest rate curve). As the model contains only one factor there are situations where the model reaches its limitations. In particular several situations where the results can be applied from the theoretical point of view, but are beyond the capacity of the model, are presented in Section 7. Here we apply the model to bond and swap options which rely mainly on one factor, the yield or swap rate, making the model appropriate.

Jamshidian [8] proposes an explicit formula for zero-coupon bonds and a less explicit one for coupon bearing bonds. His formula is obtained by writing the option on the bond as a portfolio of options on the coupons. The strike price of each individual option is adjusted in such a way that all of them are exercised simultaneously. This approach is also the one described by Hull $[6$, Section 21.4] and Musiela and Rutkowski [11, Section 12.3.5, p. 298].

The result we obtain for coupon bearing bonds is a generalization of the zero-coupon bond formula. There is a term for each coupon (as opposed to zero-coupon unique term) and one for the strike price. The formula is identical to Jamshidian's one for zero-coupon bonds but is more explicit for coupon bonds. To obtain the result, we impose a condition on the volatility. Ho and Lee and Hull and White volatility models satisfy those conditions.

The pricing formula we present contains a parameter that is obtained implicitly as the (unique) solution of a one dimensional equation with as many exponential terms as the number of coupons plus one. This equation with only two terms has to be solved to obtain the classical Black and Scholes formula [1]. In the Black and Scholes case it is possible to solve the equation explicitely and its solution is the well known " $d_{2}$ ".

Let $P(s, t)$ denote the price in $s$ of the zero coupon bond of maturity $t$ and final value 1 . We suppose that it satisfies the equation

$$
d P(t, u)=P(t, u)\left(r(t) d t+\nu(t, u) d W_{t}\right) .
$$

To obtain the explicit formula for coupon bearing bonds, we will also suppose that

H2: The function $\nu$ satisfies $\nu\left(s, t_{2}\right)-\nu\left(s, t_{1}\right)=f\left(t_{1}, t_{2}\right) g(s)$ for some positive function $g$.

Date: First version: 1 June 2000; This version: 25 January 2003.

Key words and phrases. Bond option, swaption, explicit formula, HJM model, one factor model, hedging.

JEL classification: G13

A modified version of this article appeared in The International Journal of Theoretical and Applied Finance, 6(1), 57-72, 2003. 
Our result applied to bonds follow (the precise hypothesis on the model are given in the next section). Note that we allow the payment of the strike price to be different (after) the expiry.

Theorem 1. Let $\theta \leq t_{0} \leq t_{1} \leq \cdots \leq t_{n}$. Consider a bond which pays $c_{i} \geq 0$ at times $t_{i}$ $(1 \leq i \leq n)$. We work in the Heath-Jarrow-Morton one factor model with a volatility structure of the form (H2). At time 0, the price of an European call on the bond with expiry $\theta$ and strike price $K$ to be paid in $t_{0}$ is

$$
\sum_{i=1}^{n} c_{i} P\left(0, t_{i}\right) N\left(\kappa+\alpha_{i}\right)-K P\left(0, t_{0}\right) N\left(\kappa+\alpha_{0}\right)
$$

where $\kappa$ is the (unique) solution of

$$
\sum_{i=1}^{n} c_{i} P\left(0, t_{i}\right) \exp \left(-\frac{1}{2} \alpha_{i}{ }^{2}-\alpha_{i} \kappa\right)=K P\left(0, t_{0}\right)
$$

and the $\alpha_{i}$ 's are the positive numbers such that

$$
\alpha_{i}^{2}=\int_{0}^{\theta}\left(\nu\left(s, t_{i}\right)-\nu(s, \theta)\right)^{2} d s .
$$

The price of an European put is given by

$$
K P\left(0, t_{0}\right) N\left(-\kappa-\alpha_{0}\right)-\sum_{i=1}^{n} c_{i} P\left(0, t_{i}\right) N\left(-\kappa-\alpha_{i}\right) .
$$

Note that when $n=1$ (one payment), the equation (1) is exactly the one to be solved to obtain the " $d_{2}$ " used in the Black and Scholes formula. In this respect the naming convention in the Black and Scholes formula is misleading, the " $d_{2}$ " comes before the " $d_{1}$ ". As it will be proved later, the solution of our equation is unique and non-degenerate, so it can be solved by standard numerical methods (like Newton-Raphson). In a different context, this type of equation is also the one to be solved to construct a continuously compounded zero-coupon curve linearly interpolated between two non standard points (for example the zero coupon curve in GBP between 5 and 7 years using semi-annual swaps requires to solve a similar equation with 4 terms).

In section 5 , we give an explicit way to compute an hedging ratio $(\Delta)$ of an option. It is then possible to compute the hedge ratio required for each option using its underlying instrument instruments.

In Section 7 we provide examples where unrealistic results are obtained by using the model beyond its limitations.

\section{MODEL AND HYPOTHESIS}

We use a model for $P(t, u)$, the price at $t$ of the zero-coupon bond paying 1 in $u$. We will describe this for all $0 \leq t, u \leq T$, where $T$ is some fixed constant.

When the discount curve $P(t,$.$) is absolutely continuous, which is something that is always the$ case in practice as the curve is constructed by some kind of interpolation, there exists $f(t, u)$ such that

$$
P(t, u)=\exp \left(-\int_{t}^{u} f(t, s) d s\right) .
$$

A model that describes $f$ and deduces $P$ from it can be seen as meaningful. The idea of HeathJarrow-Merton [3] was to exploit this property by supposing that

$$
d f(t, u)=\mu(t, u) d t+\sigma(t, u) d W_{t}
$$

for some suitable (stochastic) $\mu$ and $\sigma$.

Here we use a similar model, but we restrict ourself to non-stochastic coefficients. The exact hypothesis on the volatility we require is described by (H2). But we don't need all the technical refinement used in their paper or similar one, like the one described in [7] in the chapter on 
dynamical term structure model. So instead of describing the conditions that lead to such a model, we suppose that the conclusions of such a model are true. By this we mean we have a model, that we call a HJM one factor model, with the following properties.

Let $A=\left\{(s, u) \in \mathbb{R}^{2}: u \in[0, T]\right.$ and $\left.s \in[0, u]\right\}$. We work in a filtered probability space $\left(\Omega, F, \mathbb{P}^{\text {real }},\left(\mathcal{F}_{t}\right)\right)$. The filtration $\mathcal{F}_{t}$ is the (augmented) filtration of a one-dimensional standard Brownian motion $\left(W^{\text {real }}\right)_{0 \leq t \leq T}$.

H1: There exists $\sigma:[0, T]^{2} \rightarrow \mathbb{R}^{+}$measurable and bounded ${ }^{1}$ with $\sigma=0$ on $[0, T]^{2} \backslash A$ such that for some process $\left(r_{s}\right)_{0 \leq t \leq T}, N_{t}=\exp \left(\int_{0}^{t} r(s) d s\right)$ forms, with some measure $\mathbb{N}$, a numeraire pair ${ }^{2}$ (with Brownian motion $W_{t}$ ),

$$
\begin{aligned}
d f(t, u) & =\sigma(t, u) \int_{t}^{u} \sigma(t, s) d s d t-\sigma(t, u) d W_{t} \\
d P^{N}(t, u) & =P^{N}(t, u) \int_{t}^{u} \sigma(t, s) d s d W_{t}
\end{aligned}
$$

and $r(t)=f(t, t)$.

The notation $P^{N}(t, s)$ designates the numeraire rebased value of $P$, i.e. $P^{N}(t, s)=N_{t}^{-1} P(t, s)$. To simplify the writing in the rest of the paper, we will use the notation

$$
\nu(t, u)=\int_{t}^{u} \sigma(t, s) d s .
$$

Note that $\nu$ is increasing in $u$, measurable and bounded.

Moreover for $t>u, \nu(t, u)=0$, and so $P^{N}(t, u)$ is constant. Then $P(t, u)$ represents the discount factor from $u$ to $t$ when $t \leq u$ and is the money invested at the instantaneous rate $r_{s}$ for $u \leq s \leq t$ when $t>u$. In that way we have a model for the price after maturity and we can value bond options on bonds that are paying coupons before option expiry.

As we will apply our results to coupon bearing bonds, we define such a bond. Let $Q(t)=$ $\sum_{i=1}^{n} c_{i} P\left(t, t_{i}\right)$ where $c_{i}>0(1 \leq i \leq n)$ and $T>t_{n}>\cdots>t_{i}>\cdots>t_{1}$. The numeraire rebased bond satisfies

$$
d Q_{t}^{N}=\sum c_{i} P^{N}\left(t, t_{i}\right) \nu\left(t, t_{i}\right) d W_{t}=Q_{t}^{N} \rho_{t} d W_{t}
$$

for some adapted process $\left(\rho_{t}\right)_{0 \leq t \leq \theta}$.

Note that the bond we use in the hedging strategy is "generic". The random variable that defines the option can be based on different assets described by the model. In particular, an option on a 1 year bond can be hedged by a 10 years bond.

We us the following generic pricing theorem [7, Theorem 7.33-7.34].

Theorem 2. Let $V_{T}$ be some $\mathcal{F}_{T}^{W}$-measurable random variable. If $V_{T}$ is attainable, then the time $t$ value of the derivative is given by $V_{t}^{N}=V_{0}^{N}+\int_{0}^{t} \phi_{u} d Q_{u}^{N}$ where $\phi_{t}$ is the strategy and

$$
V_{t}=N_{t} \mathrm{E}_{\mathbb{N}}\left[V_{T} N_{t}^{-1} \mid \mathcal{F}_{t}\right] \text {. }
$$

\section{THE PROOF}

Before starting the proof of the result it-self, we prove two technical lemmas.

Lemma 1. In a HJM one factor model, the price of the zero coupon bond can be written has,

$$
P(t, u)=\frac{P(0, u)}{P(0, t)} \exp \left(-\frac{1}{2} \int_{0}^{t}\left(\nu^{2}(s, u)-\nu^{2}(s, t)\right) d s+\int_{0}^{t}(\nu(s, u)-\nu(s, t)) d W_{s}\right) .
$$

\footnotetext{
${ }^{1}$ Bounded is too strong for the proof we use, some $L^{1}$ and $L^{2}$ conditions are enough, but as all the examples we present are bounded, we use this condition for simplicity.

${ }^{2}$ See [7] for the definition of a numeraire pair. Note that here we require that the bonds of all maturities are martingales for the numeraire pair $(N, \mathbb{N})$.
} 
Proof. By definition of the forward rate and its equation,

$$
\begin{aligned}
P(t, u) & =\exp \left(-\int_{t}^{u} f(t, \tau) d \tau\right) \\
& =\exp \left(-\int_{t}^{u}\left[f(0, \tau)+\int_{0}^{t} \nu(s, \tau) D_{2} \nu(s, \tau) d s-\int_{0}^{t} D_{2} \nu(s, \tau) d W_{s}\right] d \tau\right) .
\end{aligned}
$$

Then using again the definition of forward rates and the Fubini theorem on inversion of iterated integrals, we have

$$
\begin{aligned}
P(t, u) & =\frac{P(0, u)}{P(0, t)} \exp \left(-\int_{0}^{t} \int_{t}^{u} \nu(s, \tau) D_{2} \nu(s, \tau) d \tau d s+\int_{0}^{t} \int_{t}^{u} D_{2} \nu(s, \tau) d \tau d W_{s}\right) \\
& =\frac{P(0, u)}{P(0, t)} \exp \left(-\int_{0}^{t} \frac{1}{2}\left(\nu^{2}(s, u)-\nu^{2}(s, t)\right) d s+\int_{0}^{t} \nu(s, u)-\nu(s, t) d W_{s}\right) .
\end{aligned}
$$

Lemma 2. In the HJM one factor model, we have

$$
N_{t}^{-1}=\exp \left(-\int_{0}^{t} r(s) d s\right)=P(0, t) \exp \left(\int_{0}^{t} \nu(s, t) d W_{s}-\frac{1}{2} \int_{0}^{t} \nu^{2}(s, t) d s\right) .
$$

Proof. The computation of this lemma are similar to the one of Lemma 1. By definition of $r$,

$$
\begin{aligned}
r(\tau) & =f(\tau, \tau)=f(0, \tau)+\int_{0}^{\tau} d f(s, \tau) d s \\
& =f(0, \tau)+\int_{0}^{\tau} \nu(s, \tau) D_{2} \nu(s, \tau) d s-\int_{0}^{\tau} D_{2} \nu(s, \tau) d W_{s} .
\end{aligned}
$$

Then using Fubini, we have

$$
\int_{0}^{t} r(\tau) d \tau=\int_{0}^{\tau} f(0, \tau) d \tau+\frac{1}{2} \int_{0}^{t} \nu^{2}(s, t) d s-\int_{0}^{t} \nu(s, t) d W_{s} .
$$

To obtain the explicit formula, we will also suppose that

H2: The function $\nu$ satisfies, for all $s \leq t_{1} \leq t_{2}, \nu\left(s, t_{2}\right)-\nu\left(s, t_{1}\right)=f\left(t_{1}, t_{2}\right) g(s)$ for some positive function $g$.

Note that as $\nu$ is measurable and bounded, so are $f$ and $g$. As $\nu$ is increasing in $t_{i}$ and $g$ is positive, $f\left(t_{1}, t_{2}\right)$ is positive for $t_{2}>t_{1}$. Moreover as $\nu$ is increasing in $t_{2}$, so is $f\left(t_{1}, t_{2}\right)$. This proves that the $\alpha_{i}$ form an increasing sequence, fact that is used in the proof of the main theorem.

Example: The Ho and Lee volatility model [4] and the Hull and White volatility model [5] satisfy the condition (H2). For Ho and Lee one has $\nu(s, t)=\sigma(t-s)$ and $\sigma(s, t)=\sigma$; for Hull and White one has $\nu(s, t)=(1-\exp (-a(t-s))) \sigma / a$ and $\sigma(s, t)=\sigma \exp (-a(t-s))$.

In the case of the Ho and Lee model, one has, for $t_{i} \geq \theta$,

$$
\alpha_{i}^{2}=\sigma^{2}\left(t_{i}-\theta\right)^{2} \theta
$$

and for the Hull and White model,

$$
\alpha_{i}^{2}=\frac{\sigma^{2}}{2 a^{3}}\left(e^{-a \theta}-e^{-a t_{i}}\right)^{2}\left(e^{2 a \theta}-1\right) .
$$

Example: For any $\nu$ that satisfies the condition, $\tilde{\nu}(s, t)=\tilde{g}(s) \nu(s, t)$ satisfies also the condition. So it is possible in the two previous models to introduce a short term rate volatility $\sigma$ that depends on the moment at which they are seen (turbulent period).

It is also possible to include some future events like year turn in the volatility structure. For example $\nu(s, t)=\sigma \int_{s}^{t} h(u) d u$ with $h(u)=1$ except for $u$ close to year end where $h(u)=2$ define a 
volatility structure where the forward rate for the year turn is twice as volatile as the other forward rates.

Example: The hypothesis (H2) covers also the type of volatility function described in [12]. They suppose that $\sigma(s, t)=\sigma(s, s) k(s, t)$ where $k(s, t)=\exp \left(-\int_{s}^{t} \kappa(x) d x\right)$ for some deterministic function $\kappa$. This type of volatility is a particular case of (H2) with

$$
g(s)=\sigma(s, s) \exp \left(-\int_{s}^{\theta} \kappa(x) d x\right) \text { and } f\left(t_{1}, t_{2}\right)=\int_{t_{1}}^{t_{2}} \exp \left(-\int_{\theta}^{u} \kappa(x) d x\right) d u .
$$

We are now ready to prove our main result.

Theorem 3. Let $0 \leq t_{0} \leq t_{1} \leq \cdots t_{m}-1 \leq t_{m}<t_{m}+1 \leq \cdots \leq t_{n}<T$ and $0 \leq \theta \leq t_{m}$. Consider a bond which pays $c_{i}>0$ at times $t_{i}(1 \leq i \leq n, i \neq m)$. In the a HJM one factor model, when the volatility term has the form (H2), the price of an European call of expiry $\theta$ on the bond with forward payment at time $t_{m}$ of $-c_{m}>0$ is given at time 0 , by

$$
\sum_{i=m}^{n} c_{i} P\left(0, t_{i}\right) N\left(\kappa+\alpha_{i}\right)
$$

where $\alpha_{i}$ are given by

$$
\alpha_{i}^{2}=\int_{0}^{\theta}\left(\nu\left(s, t_{i}\right)-\nu(s, \theta)\right)^{2} d s
$$

and $\kappa$ is the (unique) solution of

$$
\sum_{i=m}^{n} c_{i} P\left(0, t_{i}\right) \exp \left(-\frac{1}{2} \alpha_{i}^{2}-\alpha_{i} \kappa\right)=0 .
$$

The price of an European put is given by

$$
-\sum_{i=m}^{n} c_{i} P\left(0, t_{i}\right) N\left(-\alpha_{i}-\kappa\right) .
$$

The price of an European cash-or-nothing call with expiry $\theta$ and forward strike $-c_{m}$ paying 1 in $t_{m}$ when the forward price for settlement in $t_{n}$ is larger than $-c_{m}$ is

$$
P\left(0, t_{m}\right) N\left(\kappa+\alpha_{m}\right)
$$

where $\kappa$ is the (unique) solution of

$$
\sum_{i=m}^{n} c_{i} P\left(0, t_{i}\right) \exp \left(-\frac{1}{2} \alpha_{i}{ }^{2}-\alpha_{i} \kappa\right)=0 .
$$

The price of an European asset-or-nothing call with expiry $\theta$ and forward strike $-c_{m}$ paying the bond in $t_{m}$ when the forward price for settlement in $t_{n}$ is larger than $-c_{m}$ is

$$
\sum_{i=m+1}^{n} c_{i} P\left(0, t_{i}\right) N\left(\kappa+\alpha_{i}\right)
$$

where $\kappa$ is equal to the one of the previous case.

Remark: If $\theta=t_{m}$, the option is a normal option with expiry and payment date at $\theta$ and strike $c_{m}$.

Remark: It is possible to obtain an explicit result in the case where the $c_{i}$ don't have all the same sign or when the payoff is not a piecewise linear function of the $P\left(\theta, t_{i}\right)$ but any piecewise polynomial function of it. But in these cases, the equation for the $\kappa$ can have several solutions and the set on which we have to integrate the normal distribution will be non-connected and made of several intervals. The problem become more difficult to write but is not impossible to solve. 
Remark: If, like [2], we use the forward rate as a substitute of the future rate, i.e. we suppose that the price of a 3 months $\left(t_{1}-t_{2}\right)$ rate future is given in $t$ by

$$
100\left(1-4\left(\frac{P\left(t, t_{1}\right)}{P\left(t, t_{2}\right)}-1\right)\right)
$$

we can obtain the price of options on the future. The price of a call of maturity $\theta$ and strike $K$ on this future is given by

$$
\left(5-\frac{K}{100}\right) N(\kappa)+4 \frac{P\left(0, t_{1}\right)}{P\left(0, t_{2}\right)} \exp \left(-\frac{1}{2}\left(\alpha_{1}^{2}-\alpha_{2}^{2}\right)+\frac{1}{2} \beta\right) N(\kappa+\beta)
$$

where $\beta^{2}=\int_{0}^{\theta}\left(\nu\left(s, t_{1}\right)-\nu\left(s, t_{2}\right)\right)^{2} d s$ and

$$
\kappa=\frac{1}{\beta}\left(\frac{1}{4}\left(5-\frac{K}{100}\right) \ln \left(\frac{P\left(0, t_{2}\right)}{P\left(0, t_{1}\right)}\right)+\frac{1}{2}\left(\alpha_{1}^{2}-\alpha_{2}^{2}\right)\right) .
$$

Proof. Let $W_{t}^{\#}=W_{t}-\int_{0}^{t} \nu(s, \theta) d s$. By the Girsanov's theorem ([9, Section 4.2.2, p. 72]), $W_{t}^{\#}$ is a standard Brownian motion with respect to the probability $\mathbb{P}^{\#}$ of density

$$
\exp \left(\int_{0}^{\theta} \nu(s, \theta) d W_{s}-\frac{1}{2} \int_{0}^{\theta} \nu^{2}(s, \theta) d s\right)
$$

with respect to $\mathbb{N}$.

Note that $\int_{0}^{\theta}\left(\nu^{2}\left(s, t_{i}\right)-\nu^{2}(s, \theta)\right) d s$ can be written as

$$
\int_{0}^{\theta}\left(\nu\left(s, t_{i}\right)-\nu(s, \theta)\right)^{2} d s+2 \int_{0}^{\theta} \nu(s, \theta)\left(\nu\left(s, t_{i}\right)-\nu(s, \theta)\right) d s .
$$

By Lemma 1, we obtain

$$
P\left(\theta, t_{i}\right)=\frac{P\left(0, t_{i}\right)}{P(0, \theta)} \exp \left(-\frac{1}{2} \alpha_{i}^{2}+\int_{0}^{\theta} \nu\left(s, t_{i}\right)-\nu(s, \theta) d W_{s}^{\#}\right) .
$$

As the stochastic integral of a non stochastic function is normal ([9, Section 3.6, p 65]) and using condition (H2), we have

$$
P\left(\theta, t_{i}\right)=\frac{P\left(0, t_{i}\right)}{P(0, \theta)} \exp \left(-\frac{1}{2} \alpha_{i}^{2}-\alpha_{i} X\right)
$$

with $\alpha_{i}^{2}=\int_{0}^{\theta}\left(\nu\left(s, t_{i}\right)-\nu(s, \theta)\right)^{2} d s$ and $X$ a standard normal distribution with respect to $\mathbb{P} \#$. Note that condition (H2) is used to prove that $X$ is the same for all $i$.

Then the price of the call is, using Lemma 2 and the definition of $\mathbb{P}^{\#}$,

$$
\begin{aligned}
& \mathrm{E}_{\mathbb{N}}\left(e^{-\int_{0}^{\theta} r(s) d s}\left(\sum_{i=m}^{n} c_{i} P\left(\theta, t_{i}\right)\right)^{+}\right) \\
& =\mathrm{E}^{\#}\left(P(0, \theta)\left(\sum c_{i} \frac{P\left(0, t_{i}\right)}{P(0, \theta)} \exp \left(-\frac{1}{2} \alpha_{i}^{2}-\alpha_{i} X\right)\right)^{+}\right) \\
& =\mathrm{E}^{\#}\left(\left(\sum c_{i} P\left(0, t_{i}\right) \exp \left(-\frac{1}{2} \alpha_{i}^{2}-\alpha_{i} X\right)\right)^{+}\right) \\
& =\frac{1}{\sqrt{2 \pi}} \int_{-\infty}^{+\infty}\left(\sum c_{i} P\left(0, t_{i}\right) \exp \left(-\frac{1}{2} \alpha_{i}^{2}-\alpha_{i} y\right)\right)^{+} e^{-\frac{1}{2} y^{2}} d y .
\end{aligned}
$$

We show now that $h(y)=\sum c_{i} P\left(0, t_{i}\right) \exp \left(-\frac{1}{2} \alpha_{i}^{2}-\alpha_{i} y\right)$ is positive for $y<\kappa$.

Define

$$
A_{i}=c_{i} P\left(0, t_{i}\right) \exp \left(-\frac{1}{2} \alpha_{i}^{2}\right), \quad q_{1}(y)=\sum A_{i} \exp \left(-\left(\alpha_{i}-\alpha_{m}\right) y\right)+A_{m}
$$


and

$$
q_{2}(y)=-\sum \alpha_{i} A_{i} \exp \left(-\left(\alpha_{i}-\alpha_{m}\right) y\right)-\alpha_{m} A_{m}
$$

So

$$
h(y)=\exp \left(-\alpha_{m+1} y\right) q_{1}(y) \quad \text { and } \quad h^{\prime}(y)=\exp \left(-\alpha_{m+1} y\right) q_{2}(y) .
$$

First suppose that $t_{m}>\theta$, then $\alpha_{m}>0$. As $q_{2}$ is increasing, $\lim _{y \rightarrow-\infty} q_{2}(y)=-\infty$ and $\lim _{y \rightarrow+\infty} q_{2}(y)=-\alpha_{m} A_{m}>0$, then there exists $y_{0}$ such that $h$ is decreasing on $\left(-\infty, y_{0}\right)$ and increasing on $\left(y_{0},+\infty\right)$. As $\lim _{y \rightarrow-\infty} q_{1}(y)=+\infty$ and $\lim _{y \rightarrow+\infty} q_{1}(y)=A_{0}<0$, there exists $\kappa>y_{0}$ such that $h(\kappa)=0, h(y)>0$ on $(-\infty, \kappa)$ and $h(y)<0$ on $(\kappa,+\infty)$.

In the case where $t_{m}=\theta, \alpha_{m}=0$ and we have the same type of reasoning with $y_{0}=+\infty$.

The function $f(y)-K P(0, \theta)$ is positive if and only if $y<\kappa$. The price of the call is then

$$
\begin{aligned}
& \frac{1}{\sqrt{2 \pi}} \int_{-\infty}^{\kappa}\left(\sum c_{i} P\left(0, t_{i}\right) \exp \left(-\frac{1}{2} \alpha_{i}^{2}-\alpha_{i} y\right)\right) e^{-\frac{1}{2} y^{2}} d y \\
& \quad=\sum c_{i} P\left(0, t_{i}\right) \frac{1}{\sqrt{2 \pi}} \int_{-\infty}^{\kappa} \exp \left(-\frac{1}{2} \alpha_{i}^{2}-\alpha_{i} y\right) e^{-\frac{1}{2} y^{2}} d y \\
& \quad=\sum c_{i} P\left(0, t_{i}\right) N\left(\kappa+\alpha_{i}\right) .
\end{aligned}
$$

For the cash-or-nothing call, the price is

$$
\mathrm{E}^{\#}\left(P(0, \theta) \mathbb{1}\left(\sum_{i=m}^{n} c_{i} P\left(0, t_{i}\right) \exp \left(-\frac{1}{2} \alpha_{i}^{2}-\alpha_{i} X\right)>0\right)\right)=P(0, \theta) N(\kappa) .
$$

For the asset-or-nothing call, the price is

$$
\begin{gathered}
\mathrm{E}^{\#}\left(\sum_{i=m}^{n} c_{i} P\left(0, t_{i}\right) \exp \left(-\frac{1}{2} \alpha_{i}^{2}-\alpha_{i} X\right) \mathbb{1}\left(\sum_{i=m}^{n} c_{i} P\left(0, t_{i}\right) \exp \left(-\frac{1}{2} \alpha_{i}^{2}-\alpha_{i} X\right)>0\right)\right) \\
=\sum_{i=m}^{n} c_{i} P\left(0, t_{i}\right) \frac{1}{\sqrt{2 \pi}} \int_{-\infty}^{\kappa} \exp \left(-\frac{1}{2}\left(\alpha_{i}+y\right)^{2}\right) d y=\sum_{i=m}^{n} c_{i} P\left(0, t_{i}\right) N\left(\kappa+\alpha_{i}\right) .
\end{gathered}
$$

Remark: Suppose that $t_{m+1}=\theta$ and let

$$
K_{i}=\frac{P\left(0, t_{i}\right)}{P(0, \theta)} \exp \left(-\frac{1}{2} \alpha_{i}^{2}-\alpha_{i} \kappa\right) .
$$

Then by definition of $\kappa, \sum_{i=m+2}^{n} c_{i} K_{i}=-c_{m}$. This shows that the price of the call can be written as

$$
\sum c_{i}\left(P\left(0, t_{i}\right) N\left(\kappa+\alpha_{i}\right)-K_{i} P(0, \theta) N(\kappa)\right),
$$

i.e the price of the call on a set of coupons is equal to the price of a portfolio of options on the coupons with special strike prices. This is the approach of Jamshidian [8].

By looking at the proof, it can be verified that all the option are in the money together, i.e

$$
\begin{aligned}
& \left(\sum c_{i} P\left(0, t_{i}\right) \exp \left(-\frac{1}{2} \alpha_{i}^{2}-\alpha_{i} y\right)-K P(0, \theta)\right)^{+} \\
& =\sum c_{i}\left(P\left(0, t_{i}\right) \exp \left(-\frac{1}{2} \alpha_{i}^{2}-\alpha_{i} y\right)-K_{i} P(0, \theta)\right)^{+} .
\end{aligned}
$$

Remark: The hypothesis (H2) is only used to show that the random variable $X$ is the same for all $i$. So the hypothesis (H2) can be replaced by any other hypothesis that implies the same property. 


\section{ZERO-COUPON BOND OPTION}

Theorem 4. Let $0<\theta \leq t_{1}<t_{2}<T, b_{1}, b_{2}(1 \leq i, j \leq 2)$ be such that

$$
b_{i} \cdot b_{j}=\int_{0}^{\theta}\left(\nu\left(s, t_{i}\right)-\nu(s, \theta)\right)\left(\nu\left(s, t_{j}\right)-\nu(s, \theta)\right) d s,
$$

and $B=\left(b_{1}, b_{2}\right)$. Suppose that $B$ is of rank 2 .

In the HJM one factor model, the price of an European call of maturity $\theta$ (and strike 0) on a zero-coupon bond with maturity $t_{2}$ and forward payment at time $t_{1}$ of $K$ is given at time 0 by

$$
P\left(0, t_{2}\right) N\left(d_{2}\right)-K P\left(0, t_{1}\right) N\left(d_{1}\right)
$$

where for $\phi_{1}=+1$ and $\phi_{2}=-1$,

$$
d_{i}=\frac{1}{\left|b_{1}-b_{2}\right|}\left(\ln \left(\frac{P\left(0, t_{2}\right)}{K P\left(0, t_{1}\right)}\right)+\phi_{i} \frac{1}{2}\left|b_{1}-b_{2}\right|^{2}\right)
$$

Proof. Like in the previous proof, we have

$$
P\left(\theta, t_{i}\right)=\frac{P\left(0, t_{1}\right)}{P(0, \theta)} \exp \left(-\frac{1}{2}\left|b_{i}\right|^{2}-X_{i}\right)
$$

but now the $X_{i}$ are not perfectly correlated anymore. We have that $X=\left(X_{1}, X_{2}\right) \sim N\left(0, B^{T} B\right)$. Then for some $Y \sim N(0, I), X=B^{T} Y$.

The price of the option is given by

$$
\frac{1}{2 \pi} \int_{\mathbb{R}^{2}}\left(P\left(0, t_{2}\right) \exp \left(-\frac{1}{2}\left|b_{2}\right|^{2}-b_{2} \cdot y\right)-K P\left(0, t_{1}\right) \exp \left(-\frac{1}{2}\left|b_{1}\right|^{2}-b_{1} \cdot y\right)\right)^{+} \exp \left(-\frac{1}{2}|y|^{2}\right) d y .
$$

The integrand is positive when

$$
\left(b_{1}-b_{2}\right) \cdot y>\ln \left(\frac{K P\left(0, t_{1}\right)}{P\left(0, t_{2}\right)}\right)-\frac{1}{2}\left(\left|b_{1}\right|^{2}-\left|b_{2}\right|^{2}\right)=c .
$$

Let $a=b_{1}-b_{2}$. Computing the value of the option involves computing integrals of the form

$$
I_{i}=\frac{1}{2 \pi} \int_{a . y>c} \exp \left(-\frac{1}{2}\left|y+b_{i}\right|^{2}\right) d y .
$$

We change to variable $w=C^{T}\left(y+b_{i}\right)$ where $C=\left(\frac{a}{|a|}, \frac{a^{\perp}}{|a|}\right)$. Note that $C^{T}=C^{-1}$ and $|C|=1$. We obtain

$$
\begin{aligned}
I_{i} & =\frac{1}{2 \pi} \int_{w_{1}>\frac{1}{|a|}\left(c+a . b_{i}\right)} \exp \left(-\frac{1}{2}|w|^{2}\right) d w \\
& =\frac{1}{\sqrt{2 \pi}} \int_{-\infty}^{\frac{1}{|a|}\left(c+a \cdot b_{i}\right)} \exp \left(-\frac{1}{2} w_{1}^{2}\right) d w_{1} \frac{1}{\sqrt{2 \pi}} \int_{\mathbb{R}} \exp \left(-\frac{1}{2} w_{2}^{2}\right) d w_{2} .
\end{aligned}
$$

As the second integral is equal to 1 , we have

$$
I_{i}=N\left(\frac{1}{|a|}\left(c+a \cdot b_{i}\right)\right) .
$$

Note that

$$
c+a \cdot b_{i}=c+\left(b_{1}-b_{2}\right) \cdot b_{i}=\ln \left(\frac{P\left(0, t_{2}\right)}{K P\left(0, t_{1}\right)}\right)+\phi_{i} \frac{1}{2}\left|b_{1}-b_{2}\right|^{2} .
$$

Remark: If $A$ is of rank 1 , we can apply Theorem 3. 


\section{HedGING}

We now describe how to hedge an option on a coupon bearing bond by a portfolio of cash and a certain quantity of bond. So we describe the Delta of the option.

Theorem 5. Suppose the hypotheses of Theorem 3 are satisfied and let $S$ denote the bonds that pays $b_{i}$ in $t_{i}(0 \leq i \leq n)$. Then the hedging strategy is to hold

$$
\Delta=\frac{\sum_{i=m}^{n} c_{i} P\left(0, t_{i}\right) \nu\left(0, t_{i}\right) N\left(\kappa+\alpha_{i}\right)}{\sum_{i=0}^{n} b_{i} P\left(0, t_{i}\right) \nu\left(0, t_{i}\right)}
$$

bond $S$.

Proof. Let $P(t)=\left(P\left(t, t_{0}\right), \ldots, P\left(t, t_{n}\right)\right)$. By Theorem 3 the value of the option is

$$
V_{t}=F(P(t), t)
$$

with $F: \mathbb{R} \times \mathbb{R}^{n} \rightarrow \mathbb{R}$. We prove that $D_{i+1} F(0, P(0))=c_{i} N\left(\kappa+\alpha_{i}\right)$.

This is a consequence of the implicit function theorem [10]. Let $x_{i}=P\left(0, t_{i}\right)$. We have

$$
f(\kappa, x)=\sum_{i=m}^{n} c_{i} x_{i} \exp \left(-\frac{1}{2} \alpha_{i}^{2}-\alpha_{i} \kappa\right)=0 .
$$

As $D_{1} f(\kappa, x)=-\sum \alpha_{j} c_{j} x_{j} \exp \left(-\frac{1}{2} \alpha_{j}^{2}-\alpha_{j} \kappa\right) \neq 0$, we can apply the implicit function theorem. Then we have, for $i=m, \ldots, n$

$$
D_{i+1} F(0, x)=c_{i} N\left(\kappa+\alpha_{i}\right)+\left[\sum c_{j} x_{j} N^{\prime}\left(\kappa+\alpha_{j}\right)\right] D_{i} \kappa
$$

We can decompose $N^{\prime}\left(\kappa+\alpha_{j}\right)$ in $\frac{1}{\sqrt{2 \pi}} \exp \left(-\frac{1}{2} \alpha_{j}^{2}-\kappa \alpha_{j}\right) \exp \left(-\frac{1}{2} \kappa^{2}\right)$, so using the definition of $\kappa$ we have

$$
\begin{aligned}
D_{i+1} F(0, x) & =c_{i} N\left(\kappa+\alpha_{i}\right)+\left(\sum c_{j} x_{j} \exp \left(-\frac{1}{2} \alpha_{j}^{2}-\kappa \alpha_{j}\right)\right) \frac{1}{\sqrt{2 \pi}} \exp \left(-\frac{1}{2} \kappa^{2}\right) D_{i} \kappa \\
& =c_{i} N\left(\kappa+\alpha_{i}\right) .
\end{aligned}
$$

Using the multidimensional Itô formula, we have for $t<\theta$,

$$
\begin{aligned}
V_{t}^{N}= & F(0, P(0))+\int_{0}^{t} F(s, P(s)) d N_{s}^{-1}+\int_{0}^{t} N_{s}^{-1} D_{1} F(s, P(s)) d s \\
& +\int_{0}^{t} N_{s}^{-1} \sum_{i=1}^{n} D_{i+1} F(s, P(s)) d P\left(s, t_{i}\right) \\
& +\frac{1}{2} \int_{0}^{t} N_{s}^{-1} \sum_{i, j=0}^{n} D_{i+1, j+1} F(s, P(s)) d\left\langle P^{N}\left(., t_{i}\right), P^{N}\left(., t_{j}\right)\right\rangle_{s} \\
= & F(0, P(0))+\int_{0}^{t} \sum_{i=0}^{n} P^{N}\left(s, t_{i}\right) \nu\left(s, t_{i}\right) D_{i+1} F(s, P(s)) d W_{s}+\int_{0}^{t} H_{s} d s .
\end{aligned}
$$

As $V_{t}^{N}$ is a martingale under $\mathbb{N}$, we have $H_{s}=0$. By expliciting the value of the volatility of $S$ and the value of $D_{i+1} F$,

$$
V_{t}^{N}=F(0, P(0))+\int_{0}^{t} \frac{\sum_{i=m}^{n} c_{i} P^{N}\left(s, t_{i}\right) \nu\left(s, t_{i}\right) N\left(\kappa+\alpha_{i}\right)}{\sum_{i=0}^{n} b_{i} P^{N}\left(s, t_{i}\right) \nu\left(s, t_{i}\right)} d S_{s}^{N} .
$$

Theorem 2 is valid for any hedging instruments, in particular for $S$. Using this theorem and the uniqueness of the decomposition of an Itô process, we have

$$
\Delta_{t}=\phi_{t}=\frac{\sum_{i=m}^{n} c_{i} P\left(t, t_{i}\right) \nu\left(t, t_{i}\right) N\left(\kappa+\alpha_{i}\right)}{\sum_{i=0}^{n} b_{i} P\left(t, t_{i}\right) \nu\left(t, t_{i}\right)}
$$




\section{Application}

We apply our result to obtain the price of a swaption (or option on forward starting swap if we want). A receiver swaption with strike rate $R$ and expiry $\theta$ on a swap of starting date $t_{0}$ and term $t_{n}-t_{0}$ is the right to enter at time $\theta$ in a swap with starting date $t_{0}$ and maturity $t_{n}$ where one pays the floating rate and receives a fixed rate $R$. We suppose that the dates of the fixed coupons are $t_{i}>\theta(i=1, \ldots, n)$ and that the coupons are $\delta_{i} R$ with $\delta_{i}$ the accrual factors. The floating leg has a value of 1 . The swaption is equivalent to a call option on a bond paying a coupon of $R$ with strike price of 1 .

Theorem 6. Suppose we work in the HJM one factor model with a volatility term of the form (H2). We use the notation $c_{i}=\delta_{i} R(1 \leq i \leq n)$ and $c_{n}=1+\delta_{n} R$. The price of an European receiver swaption, with expiry $\theta$ and strike rate $R$ on a swap with starting date $t_{0}$ and maturity date $t_{n}$, is given at time 0 by

$$
\sum_{i=1}^{n} c_{i} P\left(0, t_{i}\right) N\left(\kappa+\alpha_{i}\right)-P\left(0, t_{0}\right) N\left(\kappa+\alpha_{0}\right)
$$

where $\kappa$ is the (unique) solution of

$$
\sum_{i=1}^{n} c_{i} P\left(0, t_{i}\right) \exp \left(-\frac{1}{2} \alpha_{i}{ }^{2}-\alpha_{i} \kappa\right)=P\left(0, t_{0}\right) \exp \left(-\frac{1}{2} \alpha_{0}{ }^{2}-\alpha_{0} \kappa\right)
$$

and

$$
\alpha_{i}^{2}=\int_{0}^{\theta}\left(\nu\left(s, t_{i}\right)-\nu(s, \theta)\right)^{2} d s .
$$

The rate of hedging $(\Delta)$ by forward swaps starting at $t_{0}$ and with fixed rate $R$ is

$$
\Delta=\frac{\sum_{i=1}^{n} c_{i} P\left(0, t_{i}\right) \nu\left(0, t_{i}\right) N\left(\kappa+\alpha_{i}\right)-P\left(0, t_{0}\right) \nu\left(0, t_{0}\right) N\left(\kappa+\alpha_{0}\right)}{\sum_{i=1}^{n} \delta_{i} R P\left(0, t_{i}\right) \nu\left(0, t_{i}\right)-P\left(0, t_{0}\right) \nu\left(0, t_{0}\right)}
$$

The hedging ratio $(\Delta)$ for swaptions or bond options is interesting in itself. It allows, at the same time, to hedge the options individually and to use a unique model for the pricing of different instruments. So the different options are priced coherently, using only one set of parameters $(\sigma$ and $a$ in the Hull and White model). But each option can be hedge by its underlying, reducing the model risk.

Remark: A method often used to compute the sensitivity of interest rate options is to differentiate the price with respect to the different rates or discount factors. This is not coherent with the model used as it is a one factor model and the rates cannot move independently. By doing that, the sensitivity to the discount factor $P\left(0, t_{i}\right)$ is $c_{i} N\left(\kappa+\alpha_{i}\right)$. The sensitivity of the underlying to the same factor is $c_{i}$. So we obtain a sensitivity of the option that is not equal to the sensitivity of a certain amount of its underlying. By this method it becomes impossible to hedge a bond option with its underlying bond, which is in contradiction with the principle of option pricing and hedging.

\section{LiMits OF THE MODEL}

The model we present is a one factor model with the factor representing intuitively the level of rates. So trades that involves several instruments or one instrument that depends of the shape of the curve can be (largely) incorrectly valued.

Section 5 explain how to hedge a bond option on the bond $Q$ with a portfolio of bond $S$ and cash. In particular an option on a one year bond could be hedged with ten year bonds. It is quite clear that the relation between the one and the ten years bonds depends largely of the shape of the curve, of its slope. So we don't advice this type of hedging strategy.

The theory does not put any restriction on the payment date with respect to the expiry (except that it has to be after). So a one year option on a ten year bond with payment seven years after the 
expiry is theoretically possible. We show now with an example that this gives gives theoretically correct results but financially aberrant.

Example: We use the times $\theta=1, t_{0}=8$, and $t_{1}=11$. We use the Hull and White model with parameter $a=0.1$ and $\sigma=0.015$ (we take those values from Example 21.2 of Hull's book [6]). The rates (continuously compounded zero-coupon) for the different dates are $r_{\theta}=4 \%, r_{0}=5 \%$, and $r_{1}=6 \%$. We study the option with expiry $\theta$ on the zero-coupon bond paying $c_{1}$ at time $t_{1}$ with a strike of $c_{0}$ to be payed in $t_{0}$. We normalize the cash flow $c_{0}$ to -1 and take $c_{1}$ such that the option is valueless is at expiry the rates are $5 \%$ and $5.5 \%$. So $c_{1}=\exp \left(-0.05\left(t_{1}-t_{0}\right)\right) / \exp \left(-0.055\left(t_{2}-t_{1}\right)\right)=$ $1.2214^{3}$. As it is a zero-coupon bond, $\kappa$ can be computed explicitely. Its value is $\kappa=-3.3456$. In our model the option is exercised only if the standard normal distribution $y$ that determines the process is such that $y<\kappa$ (see the proof of the main theorem). This happens with a probability ${ }^{4}$ of only $0.0411 \%$. The model also predict that this will happen only if at expiry the seven year rate is lower that $1.6701 \%$ and the ten year rate is lower than $3.1393 \%$.

We do the same computation with $c_{1}$ such that the option is valueless when the rates at expiry are $5 \%$ for seven and ten year (flat yield curve). The probability of exercising the option become $6.6 \mathrm{E}-8 \%$ ( 7 hundred millionth of percent). The model predicts that the exercise will take place only if the seven years rate is less than $-1-1237 \%$ and the ten years rate less than $0.6836 \%$ !

The results are strange because the value of the option depends slightly of the level of rates (that's fine) and largely of the slope of the curve between $t_{0}$ and $t_{1}$ (that's the problem).

From those discussions it is clear that if one used the model for a product that does not depend principally on one rate, some problem can appear. For standard products like a swaption or a bond option with settlement one or two days after the expiry the model gives satisfactory results.

\section{Conclusion}

We present an explicit formula for call and put options on coupon bearing bonds and swaptions in a HJM one factor model. We emphasize that care and judgment has to be used when utilizing this type of model. Being explicit, the formula is easy to implement and requires only standard functions. The only (small) difficulty is that one needs to solve a one dimensional equation involving exponential. However its solution is unique and non-degenerate and can be found by standard numerical methods. Moreover, we explain how to compute the hedging ratio $(\Delta)$ of an option, allowing to hedge each option with its underlying instrument and so reducing the model risk due to the fact the model has only one factor.

Disclaimer: The views expressed here are those of the author and not necessarily those of the Bank for International Settlements.

\section{REFERENCES}

[1] F. Black and M. Scholes. The pricing of options and corporate liabilities. Journal of Political Economy, 81:637654, 1973. 1

[2] N. Cakici and J. Zhu. Pricing eurodollar futures options with the heath-jarrow-morton model. The Journal of Futures Markets, 21(7):655-680, 2001. 6

[3] D. Heath, R. Jarrow, and A. Morton. Bond pricing and the term structure of interest rates: a new methodology for contingent claims valuation. Econometrica, 60(1):77-105, January 1992. 1, 2

[4] T. S. Y. Ho and S.-B. Lee. Term structure movements and princing of interest rate contingent claims. Journal of Finance, 41:1011-1029, 1986. 1, 4

\footnotetext{
${ }^{3}$ In the description of the results we round the number to four decimals but the exact number are used for the computations.

${ }^{4}$ This is the probability in the equivalent martingale measure we use in the proof, so not the real one. But it is the one such that the value of the option is the expected value of the payment at expiry.
} 
[5] J. Hull and A. White. Princing interest rate derivatives securities. The Review of Financial Studies, 3:573-592, 1990. 1,4

[6] J. C. Hull. Options, futures, and other derivatives. Prentice Hall, fourth edition, 2000. 1, 11

[7] P. J. Hunt and J. E. Kennedy. Financial Derivatives in Theory and Practice. Wiley series in probability and statistics. Wiley, 2000. 2, 3

[8] F. Jamshidian. An exact bond option formula. The journal of Finance, XLIV(1):205-209, march 1989. 1, 7

[9] D. Lamberton and B. Lapeyre. Introduction au calcul stochastique appliqué à la finance. Ellipses, 1997. 6

[10] J. Mawhin. Analyse: Fondements, Techniques, Evolution. De Boek, 1997. 9

[11] M. Musiela and M. Rutkowski. Martingale Methods in Financial Modelling, volume 36 of Application of Mathematics: Stochastic Modelling and Applied Probability. Springer, 1997. 1

[12] P. Ritchken and L. Sankarasubramanian. Volatility structures of forward rates and the dynamics of the term structures. Mathematical Finance, 5:55-72, 1995. 5

\section{CONTEnTs}

1. Introduction 1

2. Model and hypothesis 2

3. The proof 3

4. Zero-coupon bond option $\quad 8$

5. Hedging 9

6. Application 10

7. Limits of the model 10

8. Conclusion 11

References $\quad 11$ LAND)

Derivatives Group, Banking Department, Bank for International Settlements, CH-4002 Basel (Switzer-

E-mail address: Marc.Henrard@bis.org

$U R L:$ http://www.dplanet.ch/users/marc.henrard 